and joint involvement has become an important limitation of long term prognosis.

Severe secondary hyperparathyroidism can cause osteoarticular pain and an erosive enthesopathy which carries a risk of tendinous rupture.

Urate gout is a classical feature of terminal renal failure which usually does not persist after the onset of haemodialysis, as efficient uric acid clearance is acheived during dialysis sessions.

Periarticular calcifications are usually composed of calcium phosphate crystals and are favoured by hyperphosphataemia and other porly known factors. Deposits are frequently asymptomatic but can grow into tumoral masses or cause acute articular or periarticular flares. Bone erosions, in the vicinity of deposits can be observed. In dialysis patients, secondary oxalosis usually follows vitamin $\mathrm{C}$ supplementation of the diet and may be responsible for soft tissue calcification, articular effusion, chondrocalcinosis and a disabling finger flexor contracture.

The incidence of bone and joint infection in haemodialysis patients is 10 to 100 fold the one in the population at large, and this must remain a permanent concern as prompt diagnosis and treatment are required to improve a gloomy prognosis. The leading causative organisms are Staphylococcus species, the usual portal of entry being the arterioveinous fistula. In kidney transplant patients, very unusual pathogens can be identified.

Beta 2-microglobulin amyloidosis has been documented in a few long-standing terminal renal failure patients before the start of dialysis, but most usually develops in long term dialysis patients. Up to 65 p.cent of individuals who have received ten or more years of maintenance haemodialysis using standard cuprophane membranes are affected. Ageing is also an important risk factor. Carpal tunnel syndrome is a prominent feature, although oedema, uremic neuropathy and crystal deposition may play a role in some carpal tunnel syndromes of dialysis patients. A chronic amyloid arthropathy is also frequently observed. Features include chronic arthralgias, particularly of the shoulders, chronic joint swelling, finger tendon synovitis, large subchondral bone erosions which may lead to femoral neck fracture, and destructive arthropathy. Synovial fluid is noninflammatory and may contain numerous blood cells. Destructve spondylarthropathy predominantly affects the cervical spine and can lead to spinal cord and/or nerve root compression, usually through spine instability. Shoulder pain may be related to amyloid synovium involvement and/or amyloid thickening of the cuff tendons and subacromial bursa. Systemic deposits are rare. They can be responsible for intestinal haemorrage or cardiac dysfunction. Lack of $\beta 2$-microglobulin catabolism by the deficient kidney and unsatisfactory elimination through the dialysis membrane are important in the pathogenesis of the disorders. The use of permeable and biocompatible membranes appears to delay the onset of the disease, but has been thus far unable to totally prevent its development.

\section{SP0012 MALIGNANCIES IN RHEUMATIC DISEASES}

A Ekbom. Unit of Clinical Epidemiology, Department of Medicine, Karolinska Hospital, Stockholm, Sweden

\subsection{6/annrheumdis-2001.54}

Patients with rheumatic diseases have an increased risk overall for cancer compared to the normal population. This is most pronounced for patients with SLE, but patients with rheumatoid arthritis have also a significantly increased risk. Unpublished data from Sweden also indicate that such an association exist for patients with Morbus Bechterew as well as patients with psoriatic arthritis.

However, the increased cancer risks are not general phenomenon for cancer as such. There are distinct differences in the patterns of cancer occurrence compared to the general population. Smoking related cancers, especially lung cancer, occur in excess for both rheumatoid arthritis and SLE patients as opposed to cancers of the gastrointestinal tract (especially colorectal cancer) which are inversely associated with all four disease entities mentioned above, possibly due to the pharmacotherapy these patients are exposed to.

Hematopoetic cancers (lymphomas and leukemias) also occur more than that expected in patients with rheumatic diseases. In the case of SLE and rheumatoid arthritis these excess risks are especially prominent for lymphomas, but there is also a somewhat less pronounced increased risk for leukemias. This increased risk can probably, to some extent, be explained by immunosuppressive therapy. However, the increased risk for lymphomas in a Swedish setting was already present in these patient groups before the introduction of such therapies during the 1970's. Moreover, the risk estimates for lymphomas have not been altered after the introduction of such therapies. The absence of an impact of immunosuppressives can, perhaps, to some extent be due to a substantially increased absolute risk of lymphomas in the general population, which means that the cumulative incidence of lymphomas among patients with rheumatic disease is presently higher than it was 20 years ago.

High disease activity in patients with rheumatic arthritis seems to be associated with an even more pronounced risk for lymphomas. There are indications that anti-inflammatory therapy have a protective effect, although better data is needed before such an association can be firmly established.

In conclusion, rheumatic diseases are associated with increased risk for cancer. Hematopoetic cancers are, however, the only malignancy where a causal association has been established.

\section{SP0013 STRESS SYSTEM RESPONSE IN RHEUMATIC DISEASES}

M Cutolo. Division Rheumatology Department Internal Medicine, University of Genova, Genova, Italy

\subsection{6/annrheumdis-2001.55}

The role of stress is emerging among the factors involved in the pathophysiology of rheumatic diseases including genetic predisposition, infections and gender. A stressor is any stimulus that disturbs or threatens to disturb homeostasis. Stressors can be exogenous or endogenous and metabolic, physiologic, infectious, inflammatory, or emotional in nature. The stress system response (SSR) is the array of physiological and behavioural adaptation to restore homeostasis and is mediated through activation of the hypothalamic-pituitary- adrenal axis (HPA) and the sympathetic nervous system (SNS). Activation of HPA axis induces synthesis and release of corticotropin-releasing hormone (CRH), adrenocorticotropic hormone (ACTH and finally glucocorticoids (GC) such as cortisol. GC act in a permissive way to increase readly available energy resources to brain and heart during stress and terminate defense mechanisms to stress to prevent these responses to become destructive themself. SNS activation leads to release of norepinephrine (NE) and the sympathetic division 
of the autonomic system is associated with conferring an adaptative advantage during stressful situation.

The SSR in rheumatoid arthritis (RA) and systemic lupus erythematosus (SLE)

There is strong evidence that major life events and chronic minor stress act as important factors in RA and are associated with the onset and course of the disease. By considering that minor and major stress activate the SSR, the activation of the HPA axis is the physiologic consequence of such stimulation. However, as introduced, also inflammatory or infectious mediators such as cytokines (i.e. Il-6, TNF-alpha) are considered stressors (even with direct effects on adrenal $\mathrm{gl}$ ) and are involved in HPA axis activation. Therefore, the chronic stimulation of the HPA axis (i.e. chronic stress), as reported in early RA patients (pts) and perhaps during the course of the disease (high levels of IL-6), might explain the lover than expected HPA axis function as showed by low levels of cortisol and dehydroepiandrosterone (DHEA). However, the administration of GC replaces the hormonal hypoproduction of the exhausted adrenal glands (gl), as well as decreases the levels of the inflammatory (chronic/acute) stressors (i.e. cytokines) in RA pts. Almost $50-80 \%$ of SLE pts believe that stress had provoked their illness and high level of life stress precedes flares of the disease. As observed in RA, low levels of cortisol and DHEA have been found in SLE pts further suggesting an altered HPA axis function.

The SSR in fibromyalgia syndrome (FM) and in polymyalgia rheumatica (PMR)

Minor stress and emotional trauma may act as provoking factors in FM and may modulate the course of the disease. In particular, the evidence suggest that FM represents pathology developing from a structurally dysregulated SSR which may result from a detrimental cascade of negative neuroendocrine and behavioural reaction patterns. Pain is the most prominent complain in FM. Pain memory and amplification as well as chronic pain in itself, become a main stressor in FM pts and there can be little doubt that FM is associated with chronic stress.

Recent data suggest that FM is related to a neuroendocrine disorder characterised by hyperreactive ACTH release and a relative hyporesponse of the adrenal gl. The presence of mild hypocortisolism with a reduced adrenal responsiveness in face of an exaggerated endogenous ACTH upon HPA axis stimulation, suggest a mild feedback resistance to cortisol at the central level and support distinct disturbance in the SSR in FM. The abrupt onset of PMR, with symptoms reminding the steroid withdrawal syndrome or adrenal insufficiency (i.e. myalgia, malaise, pain, depression, etc), might be induced by intense stimulation and inefficient response of the HPA axis, including different acute stresses (i.e. surgery, infections, severe interpersonal stress) in elderly people that are ageing-predisposed to an unadeguate SSR. Our studies have shown that the reduced production of adrenal hormones (i.e. cortisol, DHEAS) in recent- onset and untreated PMR pts, seems mainly related to altered intraadrenal responsiveness to ACTH stimulation (i.e. increased 17-OHP).

\section{Therapy intervention and surgery in R. D. - Thursday 14 June, 10.00-11.45/Club A-B}

\author{
SP0119 PHYSICAL EXERCISE IN RECENT RHEUMATOID \\ ARTHRITIS
}

A Häkkinen. Department of Rehabilitation, Central Hospital, Jyväskylä, Finland

10.1136/annrheumdis-2001.56

The impact of arthritis on the individual is multidimensional and various rehabilitation programs have therefore been developed for these patients. The long-term outcome varies from a minimal loss of function to a shortened life expectancy. Although RA patients represent only $8 \%$ of all patients with musculo-skeletal diseases in Finland, they consume $40 \%$ of all hospital days used by this patient group making RA a very expensive sickness. Several studies conducted worldwide have shown that more than a half of the patients become work disabled during the first 10 years of disease. Therefore, due to the limited availability of resources, it seems that efforts should be targeted to preventive rehabilitation at the early phases of disease.

The patients with early RA have significantly lower muscle strength levels (as much as 45\% in knee extension and 20\% in grip strength) compared to healthy persons even at the time of diagnosis of RA. ${ }^{1}$ Heightened disease activity, nerve function impairment, changes in muscle metabolism, decreased muscle blood flow, degeneration of muscle fibres and disuse of the musculo-skeletal system have been suspected as possible mechanisms for muscular weakness and a loss of physical fitness. An accelerated loss of bone mineral density (BMD) leading to osteoporosis is also regarded as a common clinical problem. Even the early RA patients with high disease activity and glucocorticoids have an increased risk of osteoporosis. ${ }^{2}$

According the review of Van den Ende et al., ${ }^{3}$ dynamic exercise therapy increases aerobic capacity and muscle strength in patients with a longer duration of RA. In our studies prolonged dynamic strength training for several months (with progressively increasing loads from $40-50 \%$ to $70-80 \%$ of maximum strength) increased the muscle strength values of early RA patients back to the level of healthy subjects. ${ }^{4}$ The large increase in the knee extension strength was accompanied by a significant enlargement in the cross-sectional area of the quadriceps femoris muscle. The data demonstrate that systematic resistance training can lead, not only to functional, but also to some extent structural adaptations in the neuromuscular system of early RA patients. Furthermore, the strength gains were obtained with no detrimental effects on disease activity or joint erosions. Physical training leads to significant increases in muscle strength and physical function, but it imposes only minor effects on BMD in spine and femoral neck. The "smooth" movements in supine, sitting or standing positions or non weight-bearing exercises, as generally recommended for RA patients, do not generate such ground reaction forces on the skeleton required to increase BMD. However, the physical activity on a certain level must be continuous, because it is known that muscle strength, aerobic capacity or BMD levels obtained by various type of training will be lost during the detraining. ${ }^{5,6,7}$

The findings concerning the exercise interventions support unanimously the assumption that patients with RA can safely use individually tailored dynamic strength training or aerobic exercises to increase/maintain their muscle strength and overall physical condition without exacerbating the disease. The training to 